\title{
Should we implant a permanent pacemaker in patients with left bundle branch block and PQ prolongation following transcatheter aortic valve implantation?
}

\author{
Krzysztof Błaszyk, Anna Komosa, Marek Grygier, Anna Olasińska-Wiśniewska, Aleksander Araszkiewicz, \\ Maciej Lesiak
}

Department of Cardiology, Poznan University of Medical Sciences, Poznan, Poland

Adv Interv Cardiol 2017; 13, 1 (47): 78-81

DOI: https://doi.org/10.5114/aic.2017.66192

\begin{abstract}
Introduction
Transcatheter aortic valve implantation (TAVI) includes the risk for development of heart block. The presence of left bundle branch block (LBBB) after TAVI with $\mathrm{PQ}$ interval prolongation is not clearly defined as an indication for pacemaker implantation.
\end{abstract}

\section{Case report}

A 76-year-old woman with a combined aortic valve disease, with a predominance of severe aortic stenosis, who had been previously disqualified from the classical cardiac surgery of aortic valve replacement because of the high risk of the procedure, was qualified for transcatheter aortic valve implantation (TAVI) due to increasing symptoms of heart failure. Moreover, she had a history of long-term arterial hypertension, symptomatic heart failure (NYHA II/III), rheumatoid arthritis (RA) and degeneration of the spine in the thoracic-lumbar part and lumbar scoliosis limiting her mobility. Baseline ECG revealed: regular sinus rhythm $70 \mathrm{bpm}$, the mean electrical axis in the frontal plane $=$ ' $-20^{\circ}, \mathrm{PQ}$ interval $=170 \mathrm{~ms}$, $\mathrm{QRS}=98 \mathrm{~ms}, \mathrm{QTc}=410 \mathrm{~ms}$. The baseline echocardiography examination revealed left ventricular (LV) hypertrophy (LV $=42 \mathrm{~mm}$, posterior wall (PW) of $L V=14 \mathrm{~mm}$, interventricular septum (IVS) = $15 \mathrm{~mm}$ ), significantly stenotic, extremely calcified aortic valve (peak pressure gradient $(P P G)=174 \mathrm{~mm} \mathrm{Hg}$, mean PG (MPG) = $101.2 \mathrm{~mm} \mathrm{Hg}$, and $V \max =6.6 \mathrm{~m} / \mathrm{s}$ ) with good ejection fraction (EF) of the LV (EF - 65\%). Computed tomography scans disclosed multiple calcifications of the aortic valve (Figure $1 \mathrm{~A})$ and within the coronary sinus area (aortic valve area $417 \mathrm{~mm}^{2}$, perimeter $23.4 \mathrm{~mm}$ ). Operative mortality was estimated using the following scoring sys- tems: Euro SCORE standard $=7$ p., logistic [\%] $=6.19 \%$, EUROSCORE II $=2.11 \%$, STS score mortality [\%] $=11.12 \%$, mortality \& morbidity $=58.526$. The TAVI was carried out in general anesthesia from a femoral approach using the Prostar system. Before valve implantation balloon aortic valvuloplasty using Numed Z-Med II-X $18 \mathrm{~mm} \times 50 \mathrm{~mm}$ was performed. Following that a CoreValve $29 \mathrm{~mm}$ was implanted (the depth of implantation calculated on computed tomography scan was $4 \mathrm{~mm}$ ), which resulted in a complete elimination of the transvalvular gradient with a trace of paravalvular aortic regurgitation. There were no complications of the procedure.

On the first day after TAVI the patient's general condition was good. ECG revealed: regular sinus rhythm 86 bpm, new onset of left bundle branch block (LBBB) with QRS complex prolongation from 96 to $173 \mathrm{~ms}$ (Figure 2 A) and $\mathrm{QTc}=465 \mathrm{~ms}$. For the next 5 days the patient remained in a good general condition. Physical rehabilitation started on the second day after the TAVI procedure. Continuous ECG monitoring was applied throughout the patients' stay in the cardiac intensive care unit. On the $6^{\text {th }}$ day an episode of pre-syncope (lightheadedness and dizziness lasting approximately 15 to $20 \mathrm{~s}$ ) occurred. Its mechanism remained unknown since the patient had unplugged the ECG while going to a rest room. The ECG showed: regular sinus rhythm $64 \mathrm{bpm}$, with PQ interval prolongation to $194 \mathrm{~ms}$, LBBB with QRS complex prolongation to $173 \mathrm{~ms}$ (Figure $2 \mathrm{~A}$ ) and QTc $=473 \mathrm{~ms}$. The $24 \mathrm{~h}$ Holter-ECG monitoring did not reveal any other arrhythmias or conduction abnormalities. On the $7^{\text {th }}$ day 2 more pre-syncope episodes occurred (during normal activities, with no ECG tracing). The patient complained of a short onset of heart palpitation prior to the pre-syncope episode. Lacking ECG documentation of these episodes and

\section{Corresponding author:}

Prof. Krzysztof Błaszyk MD, PhD, Department of Cardiology, Poznan University of Medical Sciences, 1/2 Długa St, 61-848 Poznan, Poland, phone: +48 602222 143, fax: +48 6185490 94, e-mail: kblaszyk5@gmail.com

Received: 23.09.2016, accepted: 10.11.2016. 

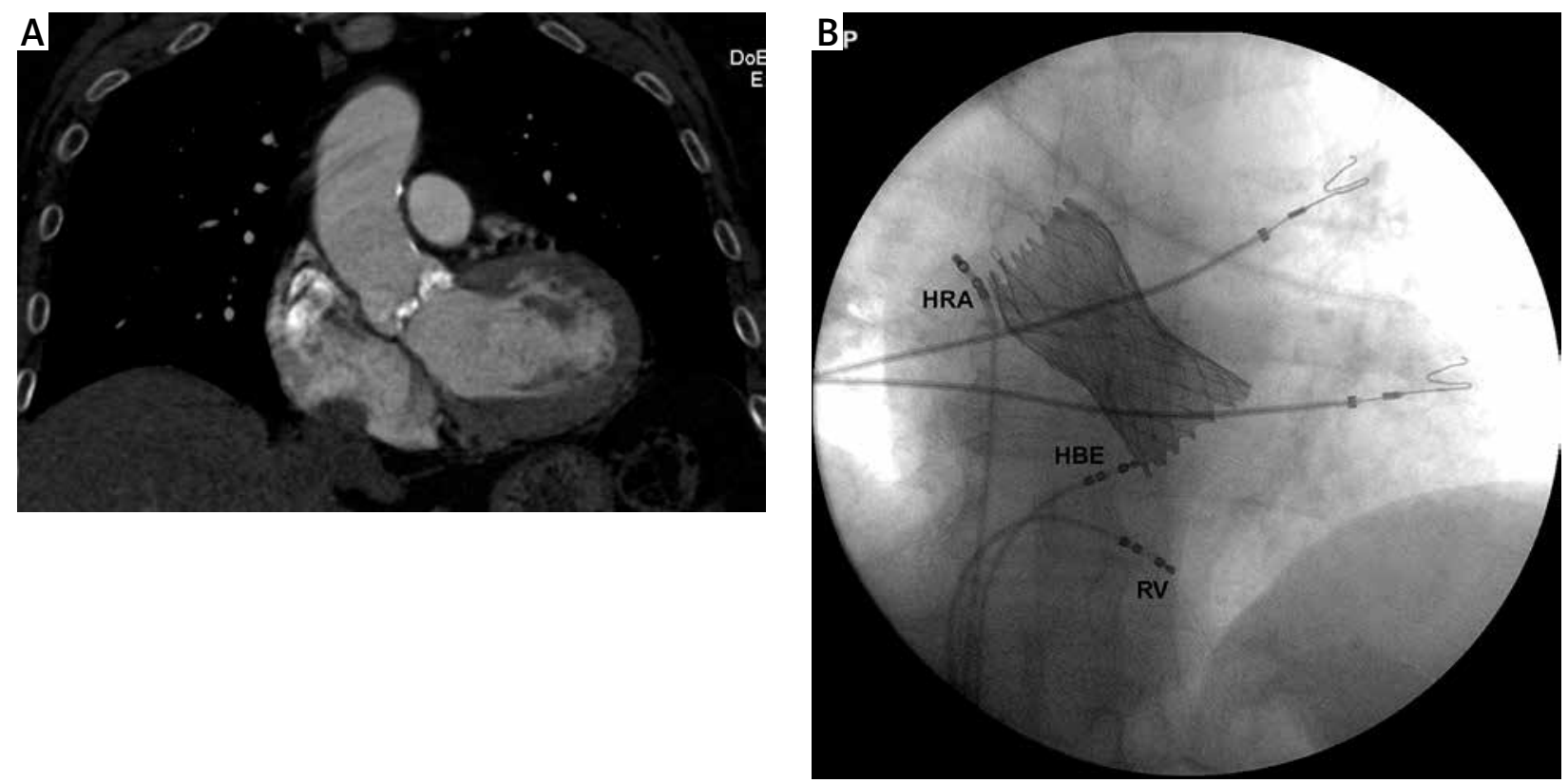

Figure 1. A - Computed tomography scan of ascending aorta with calcified aortic valve. B - Chest X-ray examination of the heart in anterior-posterior projection; CoreValve prosthesis of aortic valve and quadripolar, diagnostic catheters during electrophysiological study located on the high right atrium (HRA), in the His area $(\mathrm{HBE})$ and in the apex of the right ventricle (RV) are visualized

considering the patient's safety, the decision of invasive electrophysiology (EP) testing was made. The EP was performed on the $8^{\text {th }}$ day after TAVI, after obtaining the patient's informed consent. Three quadripolar, diagnostic catheters were used with the diameter of $6 \mathrm{Fr}$ located on the right atrium (HRA), in the His area (HBE) and in the apex of the right ventricle (RV) - Figure $1 \mathrm{~B}$. Baseline ECG during EP: sinus rhythm with LBBB. PQ interval varied and ranged from 205 to $220 \mathrm{~ms}, \mathrm{QRS}=173 \mathrm{~ms}$ (LBBB), $\mathrm{QTc}=494 \mathrm{~ms}, \mathrm{RR}=1000 \mathrm{~ms}$, AH interval = $128 \mathrm{~ms}, \mathrm{HV}$ interval $=62 \mathrm{~ms}$. Right ventricle stimulation $(\mathrm{PCL} 800+\mathrm{S} 2$ and $700+52$ ) with no retrograde conduction. Effective refractory period $\mathrm{RV}=300 \mathrm{~ms}$. Each stimulation of the right atrium resulted in an immediate progression of $A V$ block to advanced second-degree AV block. During EP examination a variable $\mathrm{PQ}$ interval was observed (with maximum prolongation to $318 \mathrm{~ms}$ ) and $\mathrm{HV}$ interval from 60-70 ms to $160 \mathrm{~ms}$. Having positioned the electrodes towards the apex of the right ventricle and His area (HBE) a dynamic increase of infra-Hisian and advanced second-degree AV block occurred with AV conduction 11 : 1 ; $6: 1$ (advanced second-degree AV block with maximal RR interval up to 10.4s) - Figure 2 B. Apart from LBBB multilevel AV conduction disturbances were found, with domination of advanced AV distal-type block. No arrhythmias were induced during the electrophysiological study.

After the EP study the patient was secured with an electrode for temporary pacing and underwent a DDD pacemaker implantation on the $8^{\text {th }}$ day after TAVI. The patient's rehabilitation and recovery on subsequent days were uneventful. Follow-up at 1 month and 6 months in the outpatient clinic revealed $99 \%$ up to $100 \%$ of right ventricular pacing.

\section{Discussion}

Here we present the analysis of clinical progression of the ECG changes in a patient after TAVI (CoreValve) who had a pre-syncope episode on the $6^{\text {th }}$ and $7^{\text {th }}$ days after valve replacement. It was necessary to make a decision whether to implant a pacemaker and, if so, when to do it. Although the literature data suggest that LBBB occurrence after TAVI is frequent (14-83\%) [1], there are no clear criteria relating to the optimal time of elective pacemaker implantation and advisability of this procedure. The presence of right bundle branch block (RBBB) before TAVI is a factor correlating with an increased risk for pacemaker implantation [2]. RBBB, however, was not present in our case. According to the literature, patients who received CoreValve and Lotus devices have higher risk of AV conduction disturbances requiring pacemaker implantation (19.2\% to $42.5 \%$ ) in comparison to the Sapien valve $(1.8 \%$ to $8.5 \%)[3,4]$.

The presence of LBBB after TAVI is a common finding, and LBBB coexisting with $\mathrm{PQ}$ interval prolongation is not clearly defined as an indication for PM implantation. The EP testing revealed a vast area of heart tissues with damaged electrophysiological properties causing AV conduction abnormalities such as multilevel AV block, possibly developing into a third-degree AV block. Probably, the mechanism of AV conduction disorders is complex. Any direct mechanical trauma or compression to the His 
A

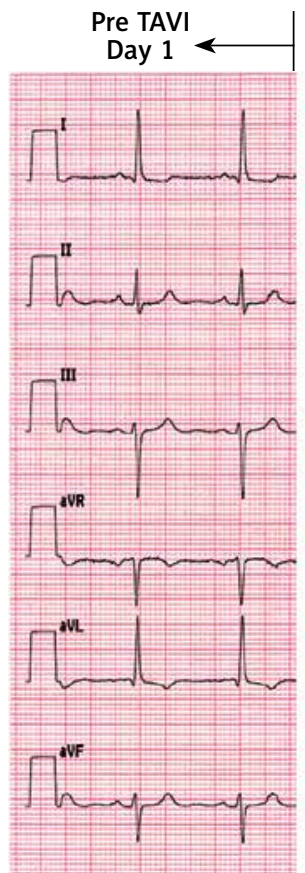

$25 \mathrm{~mm} / \mathrm{s}$

$\mathrm{PQ}=156 \mathrm{~ms}$

$\mathrm{QRS}=96 \mathrm{~ms}$
After TAVI

Day 6

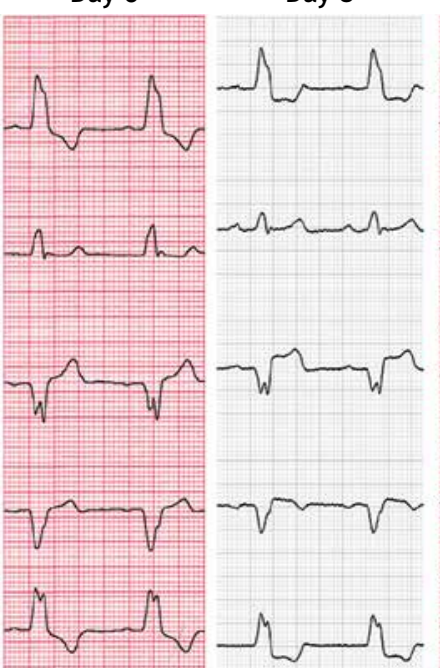

\section{EP study}

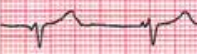

$\sim \sim^{2} r$

$\begin{array}{ll}P Q=194 \mathrm{~ms} & \mathrm{PQ}=220 \mathrm{~ms} \\ \mathrm{QRS}=173 \mathrm{~ms} & \mathrm{QRS}=173 \mathrm{~ms}\end{array}$

$\mathrm{QRS}=173 \mathrm{~ms}$

LBBB

LBBB

Syncope
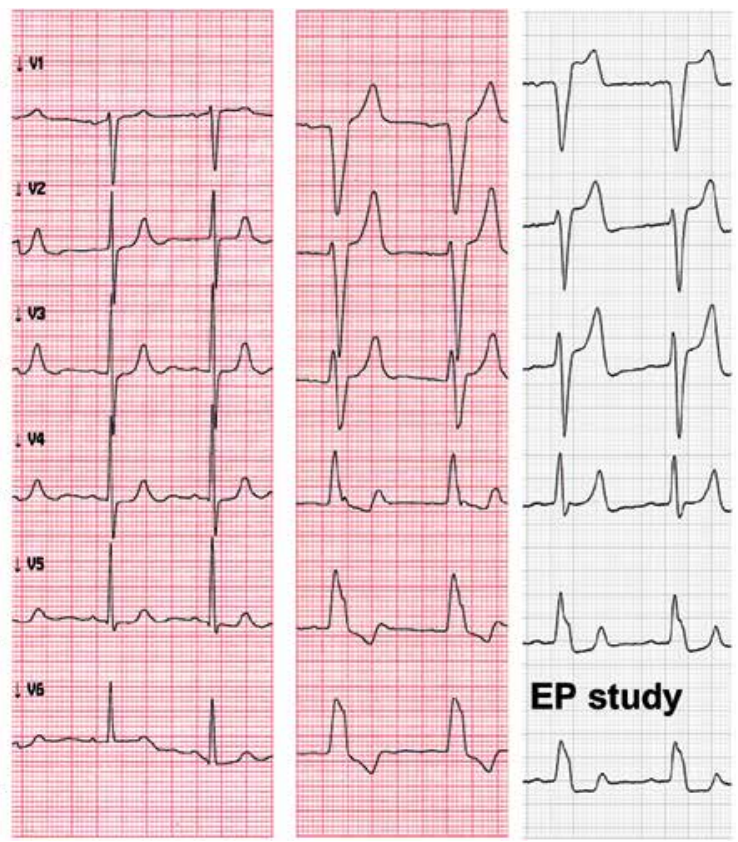

EP study

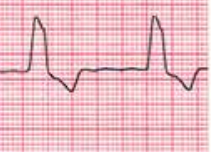

hrma

B EP study; advanced second-degree AV block (11:1) with the GAP $10.4 \mathrm{~s}$.

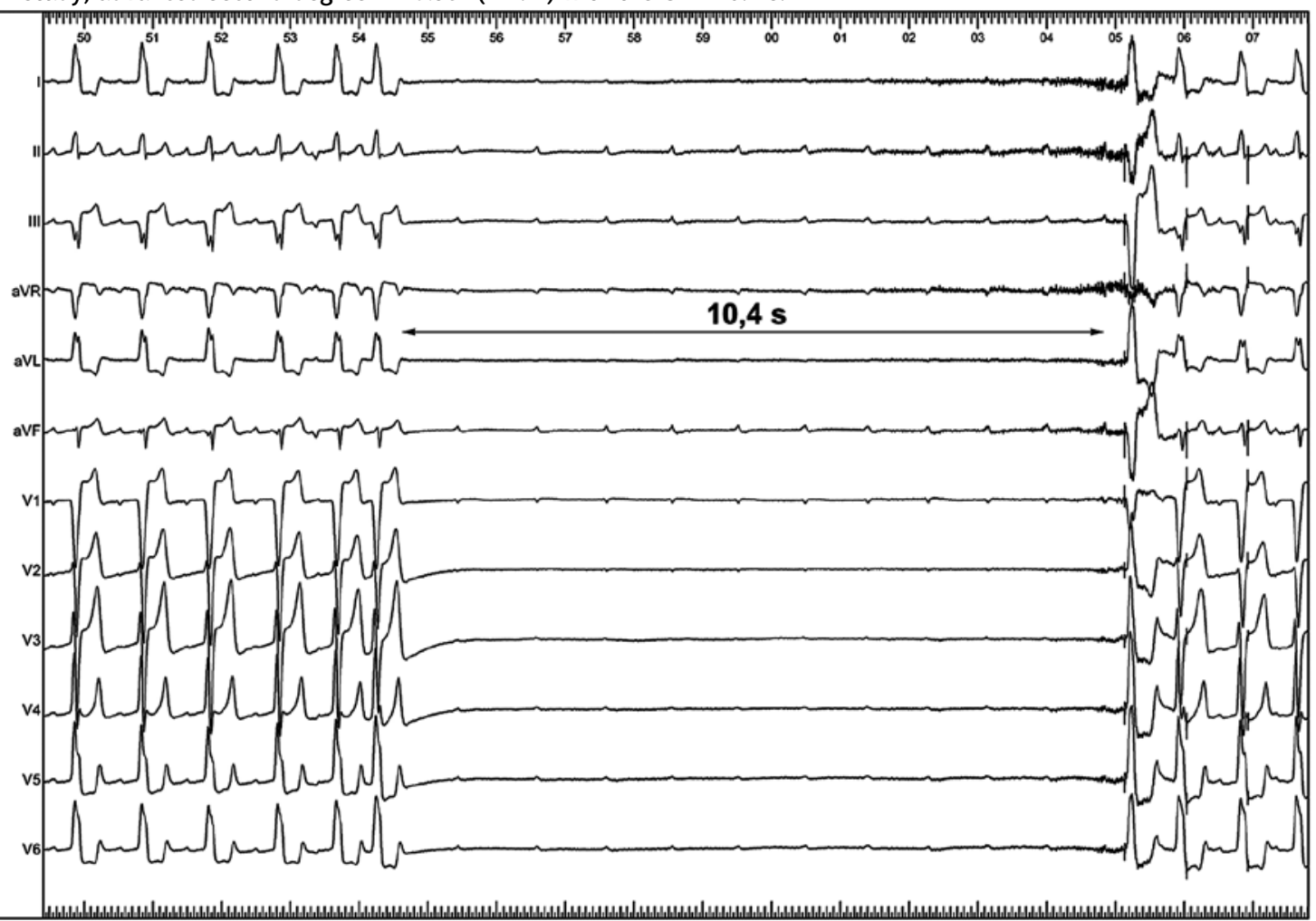

EKG speed $13 \mathrm{~mm} / \mathrm{s}$

Figure 2. A - Electrocardiogram (paper speed $25 \mathrm{~mm} / \mathrm{s}$ ) before transcatheter aortic valve implantation (TAVI), on days 6 and 8 after TAVI; left bundle branch block progression with PQ interval prolongation to 194 ms on day 6 . Also, with a more intense prolongation of PQ interval to $220 \mathrm{~ms}$ followed by an episode of syncope (day 8). B - ECG (paper speed $13 \mathrm{~mm} / \mathrm{s}$ ) spontaneous advanced second-degree atrioventricular block with 10.4 s gap 
bundle related to a self-expandable valve prosthesis in the region of the membranous septum and right trigone beneath the noncoronary/right coronary cups and the area of the upper $1 / 3$ of the intra-ventricular septum needs to be considered. Significant calcification of aortic valve leaflets may also contribute to conduction disturbances. Therefore, the optimal valve implantation in the LVOT above the His bundle is crucial. The location of the His bundle and its distal part with branches in the intraventricular septum near the left ventricle endocardium is another important issue. It can be assumed that severe concentric hypertrophy of the LV (the dimension of IVS) distinctive in aortic stenosis, in patients with good $E F$, may affect the conductive system at various levels. Eventually, the insertion of diagnostic electrodes for the EP testing may also contribute to exertion of mechanical pressure on tissues. However, dynamic progression of AV block is not observed in routine EP examination.

In this case, the EP testing was a useful tool to take the final decision to implant a pacemaker. However, in our opinion, this strategy may be used in selective cases in the early period after TAVI to reduce the number of unnecessary pacemaker implantations. According to the statement from the 2013 ESC Guidelines on cardiac pacing and cardiac resynchronization therapy [5]:

1. An intrinsic AV block should be differentiated from the other known forms of the block, namely, vagal (extrinsic) and idiopathic AV block. The infra-Hisian block by EPS supports a diagnosis of intrinsic AV block.

2. The presence of bundle branch block (BBB), unexplained syncope and non-diagnostic investigations; pacing may be considered in selected patients with unexplained syncope and BBB (class II b, level B).

3. Asymptomatic BBB; pacing is not indicated for BBB in asymptomatic patients (class III, level B).

Therefore, in such specific clinical situations, a question arises: what is the proper action? An individual approach to each patient seems to be the best answer.

\section{Conclusions}

In patients after the TAVI procedure (particularly with CoreValve) in the presence of LBBB and prolonged PQ interval, EP study may be considered to establish the indication for PM implantation.

\section{Conflict of interest}

The authors declare no conflict of interest.

\section{References}

1. Holmes DR Jr, Mack MJ, Kaul S, et al. 2012 ACCF/AATS/SCAI/ STS expert consensus document on transcatheter aortic valve replacement. J Am Coll Cardiol 2012; 59: 1200-54.

2. Adams DH, Popma JJ, Reardon MJ, et al. Transcatheter aortic-valve replacement with a self-expanding prosthesis. N Engl J Med 2014; 370: 1790-8.
3. Meredith Am IT, Walters DL, Dumonteil N, et al. Transcatheter aortic valve replacement for severe symptomatic aortic stenosis using a repositionable valve system: 30-day primary endpoint results from the REPRISE II study. J Am Coll Cardiol 2014; 64: 1339-48.

4. Popma JJ, Adams DH, Reardon MJ, et al. Transcatheter aortic valve replacement using a self-expanding bioprosthesis in patients with severe aortic stenosis at extreme risk for surgery. J Am Coll Cardiol 2014; 63: 1972-81.

5. Brignole M, Auricchio A, Baron-Esquivias G, et al. 2013 ESC Guidelines on cardiac pacing and cardiac resynchronization therapy: the Task Force on cardiac pacing and resynchronization therapy of the European Society of Cardiology (ESC). Developed in collaboration with the European Heart Rhythm Association (EHRA). Eur Heart J 2013; 34: 2281-329. 